\title{
Consonant Continuous Belief Functions Conflicts Calculation
}

\author{
Jean-Marc Vannobel \\ LAGIS \\ Université Lille1, Sciences et Technologies \\ 59655 Villeneuve d'Ascq Cedex, France \\ jean-marc.vannobel@univ-lille1.fr
}

\begin{abstract}
Much information sources model imprecision by the way of unimodal, consonant and continuous probability density functions (pdfs). We consider here in the framework of belief functions on real numbers, agents of evidence deduced from such pdfs. First are singletons plausibilities in conjunctive and disjunctive combinations proposed to basically merge agents of evidence with consonant focal elements. Second are partial and global conflict calculation methods provided. An application shows the plausibility curves and conflict values obtained in case of combination operations done on Gaussian based agents and at last, an example of conflict management based on an RCR-S adaptive rule of combination is given.
\end{abstract}

Key words: continuous belief functions. Conjunctive and disjunctive combination. Partial and global conflict calculation.

\section{Introduction}

The focal elements of agents of evidence obtained from consonant pdfs can be ordered and thus labeled by a continuous index. Under the assumption of cognitive independence between agents, this is useful to simplify expressions of the conjunctive [1] and disjunctive rule of combination [2]. As we will see, this also helps to calculate the partial and global conflict weights when much pieces of evidence are merged.

\section{Characteristics of focal intervals}

\section{$2.1 \quad$ Focal intervals}

Let $f$ be a continuous unimodal and consonant pdf of mode $\mu$ and support $\Omega=\left[\Omega^{-}, \Omega^{+}\right]$with bounds in $\mathcal{R}$, the extended set of real numbers [1]. Focal elements of the piece of evidence $\mathcal{E}$ based on $f$ are nested intervals that we label according to their fitting order by an continuous index called $z$. These intervals correspond to $A^{z}=\left[A^{z-}, A^{z+}\right], A^{z-} \in\left[\Omega^{-}, \mu\right], A^{z+} \in\left[\mu, \Omega^{+}\right]$and are deduced from the pdf by horizontal cuts such that $f\left(A^{z-}\right)=f\left(A^{z+}\right)$ in case of 'bell shaped' pdfs [1]. 


\subsection{The $\mathrm{z}$ index}

Suppose that the focal elements bounds $A^{z-}$ and $A^{z+}$ are defined according to the distances to the pdf's mode $\Delta^{-}(z) z$ and $\Delta^{+}(z) z$ such that:

$$
A^{z}=\left[\mu-\Delta^{-}(z) z, \mu+\Delta^{+}(z) z\right], z \in[0, \infty] .
$$

When $\Delta^{-}$and $\Delta^{+}$differ from 0 , the focal elements bounds are linked by a bijective function called $\gamma[1]$. This happens in case of symmetrical pdfs as illustrated in figure 1 or for some triangular distributions for instance [2].

When possible, $z$ has to be expressed by a linear relation depending on the r.v $x \in \Omega$ and the pdf's parameters, giving thus constant values for $\Delta^{-}$and $\Delta^{+}$ in some occasions. Defining $z$ using the pdfs parameters provides in most cases single belief functions expressions for a same family of pdfs [2]. For Gaussian or Laplace pdfs for instance, $\Delta$ equals to the standard deviation $\sigma$ if the $z$ index is expressed by the absolute value of the standard score:

$$
z=\frac{|x-\mu|}{\sigma}, z \in \mathcal{R}^{+}=[0, \infty] .
$$

Focal intervals $A^{z}$ correspond in that case to:

$$
A^{z}=[\mu-\sigma z, \mu+\sigma z], z \in[0, \infty] .
$$

\subsection{Focal set graphical representation}

The Grey area in figure 1 illustrates the domain representing the focal intervals set $\mathcal{F}_{i}$ corresponding to a pdf $\operatorname{Bet}_{i}$ with intervals ordered according to their $z$ label value. For a Gaussian pdf and when $z$ is defined as in (2) like done by Ristic et al. [3], $\mathcal{F}_{i}$ has a triangular shape.

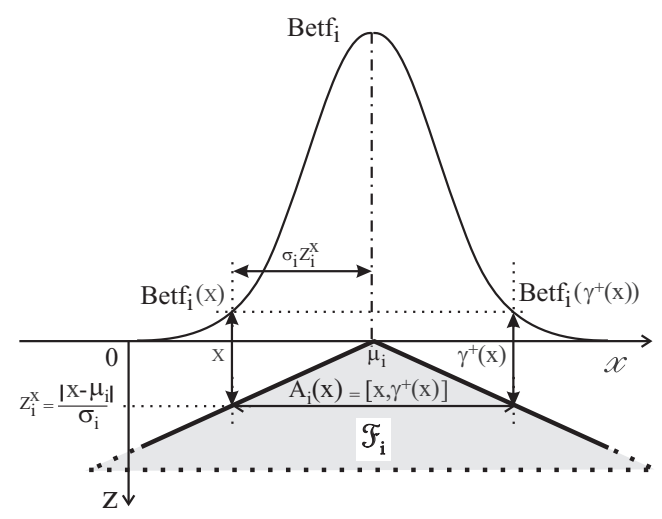

Fig. 1. Focal intervals domain $\mathcal{F}_{i}$ resulting from a Gaussian pdf. 


\section{Singleton plausibilities in combination operations of independent consonant basic belief densities}

\section{1 introduction}

We propose to construct the plausibility curve resulting of the combination of cognitively independent consonant pieces of evidence.

Suppose two such pieces of evidence $\mathcal{E}_{i}$ and $\mathcal{E}_{j}$ related to unimodal $\left(\mu_{i} \leq \mu_{j}\right)$ and consonant basic belief densities (bbd) $m_{i}$ and $m_{j}$ and at last a r.v $x$ on $\Omega=[-\infty, \infty]$. Focal sets are called $\mathcal{F}_{i}$ and $\mathcal{F}_{j}$. According to x, ordering indexes are $z_{i}^{x}$ and $z_{j}^{x}$. We note $\mathcal{F}_{i}^{x}$ and $\mathcal{F}_{j}^{x}$ the subsets of $\mathcal{F}_{i}$ and $\mathcal{F}_{j}$ that intersect with $x, \overline{\mathcal{F}_{i}^{x}}$ and $\overline{\mathcal{F}_{j}^{x}}$ their complements such that:

$$
\left\{\begin{array}{l}
\mathcal{F}_{i}=\mathcal{F}_{i}^{x} \cup \overline{\mathcal{F}_{i}^{x}}, \mathcal{F}_{i}^{x}=\left\{A \in \mathcal{F}_{i}, x \in \Omega, A \cap x \neq \emptyset\right\} \\
\mathcal{F}_{j}=\mathcal{F}_{j}^{x} \cup \overline{\mathcal{F}_{j}^{x}}, \mathcal{F}_{j}^{x}=\left\{A \in \mathcal{F}_{j}, x \in \Omega, A \cap x \neq \emptyset\right\}
\end{array}\right.
$$

\subsection{Graphical representation of combined focal sets}

Figure 2 illustrates a Venn diagram of the intervals of $\mathcal{F}_{i} \mathrm{x} \mathcal{F}_{j}$ concerned in a combination operation of $\mathcal{E}_{i}$ and $\mathcal{E}_{j}$ relatively to $x$. The whole domain represents $\mathcal{F}_{i} \mathrm{x} \mathcal{F}_{j}$ into a Cartesian coordinate system that becomes a n-dimensional space when $n$ agents have to be combined. Axes correspond to the focal sets $\mathcal{F}_{i}$ and $\mathcal{F}_{j}$, ordered in accordance to their respective $z$ labels. $\mathcal{F}_{i}^{x}, \mathcal{F}_{j}^{x}$ sets and their complements $\overline{\mathcal{F}_{i}^{x}}$ and $\overline{\mathcal{F}_{j}^{x}}$ are thus separated on axes at locations $z_{i}^{x}$ and $z_{j}^{x}$.

When focal domains have a triangular shape as illustrated in figure 1, linear relations link pairs $\left(z_{i}^{x}, z_{j}^{x}\right), x \in \Omega$ and draw the lines (1), (2) and (3) shown in figure 2. According to $z_{i}^{x}$ and $z_{j}^{x}$ expressions given for Gaussian pdfs by relation (2) and the inter-modal distance $\left|\mu_{i}-\mu_{j}\right|$, these line relations correspond to:

$$
\left\{\begin{array}{l}
\text { (1) }: z_{j}^{x}=\frac{\left|\mu_{i}-\mu_{j}\right|+\sigma_{i} z_{i}^{x}}{\sigma_{j}}, x \in\left[-\infty, \mu_{i}\right], \\
\text { (2) }: z_{j}^{x}=\frac{\left|\mu_{i}-\mu_{j}\right|-\sigma_{i} z_{i}^{x}}{\sigma_{j}}, x \in\left[\mu_{i}, \mu_{j}\right], \\
\text { (3) }: z_{j}^{x}=\frac{-\left|\mu_{i}-\mu_{j}\right|+\sigma_{i} z_{i}^{x}}{\sigma_{j}}, x \in\left[\mu_{j},+\infty\right] .
\end{array}\right.
$$

The line segment called (2) corresponds to the inter-modal interval $\left[\mu_{i}, \mu_{j}\right]$ and pairs $\left(z_{i}^{x}, z_{j}^{x}\right)$ satisfying the inequality (11) correspond to disjoint focal intervals $A_{i}^{z}$ and $A_{j}^{z}$. Relatively to the partial conflict $k_{i, j}$ existing between the agents $\mathcal{E}_{i}$ and $\mathcal{E}_{j}$, the values of the $z$ indexes $z_{i}^{\mu_{j}}$ and $z_{j}^{\mu_{i}}$ are called $K_{i, j}$ and $K_{j, i}$ such as:

$$
\left\{\begin{array}{l}
K_{i, j}=z_{i}^{\mu_{j}}=\frac{\left|\mu_{j}-\mu_{i}\right|}{\sigma_{i}}, \\
K_{j, i}=z_{j}^{\mu_{i}}=\frac{\left|\mu_{j}-\mu_{i}\right|}{\sigma_{j}}
\end{array} .\right.
$$

And we note $K i, j$ and $K j, i$ the bounds of the line segment (2) such as:

$$
\left\{\begin{array}{l}
K i, j=\left(K_{i, j}, 0\right) \\
K j, i=\left(0, K_{j, i}\right)
\end{array}\right.
$$

At last, as we can see in figure 2 , each pair $\left(z_{i}^{x}, z_{j}^{x}\right)$ separates the domain $\mathcal{F}_{i} \mathrm{x} \mathcal{F}_{j}$ in four subsets of interest as in a Karnaugh map. These subsets correspond to $\overline{\mathcal{F}_{i}^{x}} \times \overline{\mathcal{F}_{j}^{x}}, \overline{\mathcal{F}_{i}^{x}} \times \mathcal{F}_{j}^{x}, \mathcal{F}_{i}^{x} \times \overline{\mathcal{F}}_{j}^{x}, \mathcal{F}_{i}^{x} \times \mathcal{F}_{j}^{x}$. 


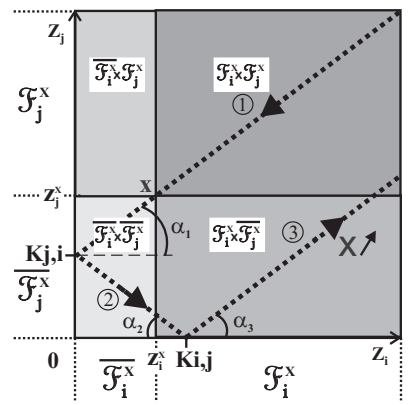

Fig. 2. Venn diagram of two Gaussian pdfs combination $\left(\mu_{i}<\mu_{j}\right)$.

\subsection{Singleton's plausibility in conjunctive and disjunctive combination of consonant bbds}

The plausibility of a singleton relatively to a piece of evidence $\mathcal{E}$ with focal elements indexed by $z$ based on a consonant and unimodal pdf is:

$$
P l(x)=P l\left(z^{x}\right)=\int_{z=z^{x}}^{z=z^{x} \max } m(z) d z=1-M\left(z^{x}\right)
$$

with $M$ the integral of the bbd $m$ and $z^{x_{\max }}$ the upper bound of $z$ 's domain.

The singletons plausibility after conjunctive combination of $\mathcal{E}_{i}$ and $\mathcal{E}_{j}\left(\mathcal{E}_{i} \perp \mathcal{E}_{j}\right)$ corresponds to:

$$
P l_{i @ j}(x)=P l_{i}\left(z_{i}^{x}\right) P l_{j}\left(z_{j}^{x}\right) .
$$

In case of the disjunctive combination of $\mathcal{E}_{i}$ and $\mathcal{E}_{j}[2]$ and as for Smets Disjunctive Rule of Combination (DRC) [5], the plausibility $P l_{i @ j}(x)$ is given by:

$$
\begin{aligned}
P l_{i @ j}(x) & =1-\left(1-P l_{i}\left(z_{i}^{x}\right)\right)\left(1-P l_{j}\left(z_{j}^{x}\right)\right), \\
& =1-M_{i}\left(z_{i}^{x}\right) M_{j}\left(z_{j}^{x}\right) .
\end{aligned}
$$

Relations (9) and (10) can be generalized to merge $n$ agents [2].

\section{Conflict calculation in case of consonant bbds}

\subsection{Partial conflict}

The domain of the partial conflict $k_{i, j}$ existing between two cognitively independent pieces of evidence $\mathcal{E}_{i}$ and $\mathcal{E}_{j}$ based on unimodal and consonant pdfs can be observed graphically. It corresponds in figure 2 to the triangle $(0 ; K i, j ; K j, i)$. The intervals concerned by this area are disjoint and in case of Gaussian pdfs for instance, satisfy:

$$
0 \leq z_{j}^{x} \leq \frac{\left|\mu_{i}-\mu_{j}\right|-\sigma_{i} z_{i}^{x}}{\sigma_{j}} .
$$

Calculation of $k_{i, j}$ following this is expressed here according to the $z$ labels and the inter-modal distance. 
Consider modes $\mu_{i}<\mu_{j}$, bounded or infinite supports $\Omega_{i}=\left[\Omega_{i}^{-}, \Omega_{i}^{+}\right]$and $\Omega_{j}=\left[\Omega_{j}^{-}, \Omega_{j}^{+}\right]$, labels $z_{i} \in\left[0, z_{\text {max }_{i}}\right], z_{j} \in\left[0, z_{\text {max }_{j}}\right]$ satisfying (1).

If $\Omega_{i} \cap \Omega_{j} \neq \emptyset$, the partial conflict $k_{i, j}$ differs from 1 and corresponds to:

$$
k_{i, j}=m_{i @ j}(\emptyset)=\int_{z_{i}=0}^{z_{i}=z_{\operatorname{Max}_{i}}} \int_{z_{j}=0}^{z_{j}=z_{\operatorname{Max}_{j}}} m_{i}\left(z_{i}\right) m_{j}\left(z_{j}\right) d z_{j} d z_{i}
$$

where:

$$
\left\{\begin{array}{l}
z_{\operatorname{Max}_{i}}=\min \left(\frac{\left|\mu_{i}-\mu_{j}\right|}{\Delta_{i}^{+}}, z_{\text {max }_{i}}\right), \\
z_{\text {Maxj }_{j}}=\min \left(\frac{\left|\mu_{i}-\mu_{j}\right|-\Delta_{i}^{+} z_{i}}{\Delta_{j}^{-}}, z_{\text {max }_{j}}\right) .
\end{array}\right.
$$

Partial conflict $k_{j, i}=k_{i, j}$ can also be calculated from the variable $z_{j}$.

Note that in case of pdfs with infinite support, relations (13) reduce to:

$$
\left\{\begin{array}{l}
z_{M^{\prime} x_{i}}=\frac{\left|\mu_{i}-\mu_{j}\right|}{\Delta_{i}^{+}}, \\
z_{M^{\prime a x}}=\frac{\left|\mu_{i}-\mu_{j}\right|-\Delta_{i}^{+} z_{i}}{\Delta_{j}^{-}} .
\end{array}\right.
$$

For symmetrical pdfs as Gaussian or Laplace ones, relations (13) become:

$$
\left\{\begin{array}{l}
z_{\operatorname{Max}_{i}}=\frac{\left|\mu_{i}-\mu_{j}\right|}{\sigma_{i}}, \\
z_{\operatorname{Max}_{j}}=\frac{\left|\mu_{i}-\mu_{j}\right|-\sigma_{i} z_{i}}{\sigma_{j}} .
\end{array}\right.
$$

Relation (12) can be reduced to a single integral by using the bbd's cumulative expression $M$ (or equivalently $1-P l$ ) [2]. The weight of conflict becomes thus:

$$
k_{i, j}=m_{i @ j}(\emptyset)=\int_{z_{i}=0}^{z_{i}=z_{\operatorname{Max}}} m_{i}\left(z_{i}\right) M_{j}\left(z_{\text {Max }_{j}}\right) d z_{i} .
$$

Since bbds resulting from pdfs are normalized, it is possible to calculate the partial conflict from intersecting intervals instead of those that do not. When $\mu_{i} \leq \mu_{j}, k_{i, j}$ corresponds to:

$$
\begin{aligned}
k_{i, j} & =1-\int_{z_{i}=0}^{z_{i}=z_{\max _{i}}} \int_{z_{j}=z_{M i n_{j}}}^{z_{j}=z_{M_{i}}} m_{i}\left(z_{i}\right) m_{j}\left(z_{j}\right) d z_{j} d z_{i} \\
& =1-\int_{z_{i}=0}^{z_{i}=z_{\max _{i}}} m_{i}\left(z_{i}\right)\left(P l_{j}\left(z_{M_{i n}}\right)-P l_{j}\left(z_{\text {Max }_{j}}\right)\right) d z_{i}
\end{aligned}
$$

with:

$$
\left\{\begin{array}{l}
z_{\operatorname{Min}_{j}}=\max \left(0, \frac{\left|\mu_{i}-\mu_{j}\right|-\Delta_{i}^{+} z_{i}}{\Delta_{j}^{-}}\right), \\
z_{\operatorname{Max}_{j}}=\max \left(z_{\max _{j}}, \frac{\left|\mu_{i}-\mu_{j}\right|-\Delta_{i}^{+} z_{i}}{\Delta_{j}^{-}}\right) .
\end{array}\right.
$$

Relations (18) are justified for $z_{M i n_{j}}$ by the fact that $\frac{\left|\mu_{i}-\mu_{j}\right|-\Delta_{i}^{+} z_{i}}{\Delta_{j}^{-}}<0$ when $A^{z_{i}+}>\mu_{j}$ and means in that case, that all the focal elements of $\mathcal{E}_{j}$ have to be considered $\left(z_{\mathrm{Min}_{j}}=0\right) . z_{\mathrm{Max}_{j}}$ 's relation takes into account the case of a bounded support pdf for $\mathcal{E}_{j}$ leading to a total conflicting situation when $A_{i}^{z_{i}} \cap \Omega_{j}=\emptyset$, corresponding thus to $z_{\mathrm{Min}_{j}}=z_{\mathrm{Max}_{j}}$.

Relations (17) and (18) can be simplified in case of infinite supports and symmetrical pdfs. 


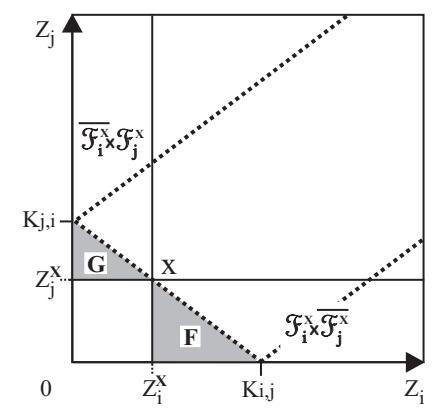

Fig. 3. Conflicting domains $F$ and $G$ included in the $\mathcal{F}_{i}^{x} \times \overline{\mathcal{F}_{j}^{x}}$ and $\overline{\mathcal{F}_{i}^{x}} \times \mathcal{F}_{j}^{x}$ sets.

\subsection{Conflict abacus for Least Committed bbds based on Gaussian pdfs}

Figure 4 presents an abacus of the partial conflict for bbds deduced from two Gaussian pdfs $\left(\mathcal{N}_{1}\left(x ; \mu_{1}, \sigma_{1}^{2}\right), \mathcal{N}_{2}\left(x ; \mu_{2}, \sigma_{2}^{2}\right)\right)$. Parameters $K i, j$ and $K j, i$ are defined using relations (6) and correspond to the bounds of the line segment (2) showed in figure 2 .

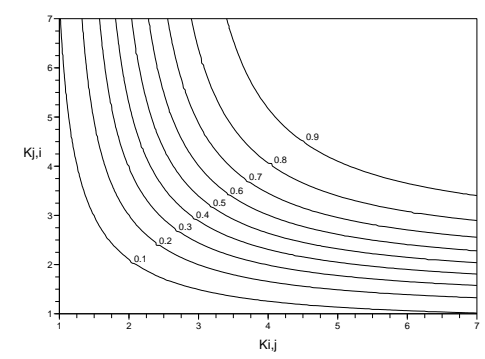

Fig. 4. Conflict's map in case of two Gaussian based agents of evidence combination.

\subsection{Conflict's part in disjunctive combination}

The disjunctive combination of two agents includes the XOR combination of sets $\mathcal{F}_{i}^{x}$ and $\mathcal{F}_{j}^{x}$. This may take into account non convex focal intervals located in the partial conflict's domain as we can see in figures 2 and 3. Singletons $x$ concerned take values in $\left[\mu_{i}-\frac{\Delta_{i}^{-}}{\Delta_{i}^{+}}\left|\mu_{i}-\mu_{j}\right|, \mu_{j}+\frac{\Delta_{j}^{+}}{\Delta_{j}^{-}}\left|\mu_{i}-\mu_{j}\right|\right]$ when $\mu_{i} \leq \mu_{j}$ and figure 3 illustrates this for $x \in\left[\mu_{i}, \mu_{j}\right]$ with the grey areas $F$ and $G$. In case of infinite 
support pdfs, $F$ and $G$ are defined as:

$$
\left\{\begin{aligned}
F(x) & =\int_{z_{i}=z_{i}^{x}}^{z_{i}=K_{i, j}} M_{i}\left(z_{i}\right) m_{j}\left(\frac{\left|\mu_{i}-\mu_{j}\right|-\Delta_{i}^{+} z_{i}}{\Delta_{j}^{-}}\right) d z_{i} & & \text { if } 0 \leq z_{i}^{x} \leq K_{i, j} \\
& =0 & & \text { otherwise, } \\
G(x) & =\int_{z_{j}=z_{j}^{x}}^{z_{j}=K_{j, i}} M_{j}\left(z_{j}\right) m_{i}\left(\frac{\left|\mu_{i}-\mu_{j}\right|-\Delta_{j}^{-} z_{j}}{\Delta_{i}^{+}}\right) d z_{j} & & \text { if } 0 \leq z_{j}^{x} \leq K_{j, i} \\
& =0 & & \text { otherwise } .
\end{aligned}\right.
$$

Figure 5 shows the degrees of conflict $F$ and $G$ taken into account in disjunctive combination of two bbds based on Gaussian pdfs. The maximum conflicting situations correspond as an evidence to singletons $\mu_{i}$ and $\mu_{j}$ and thus respectively to index pairs $\left(z_{i}, z_{j}\right)$ equal to $\left(0, K_{j, i}\right)$ and $\left(K_{i, j}, 0\right)$.

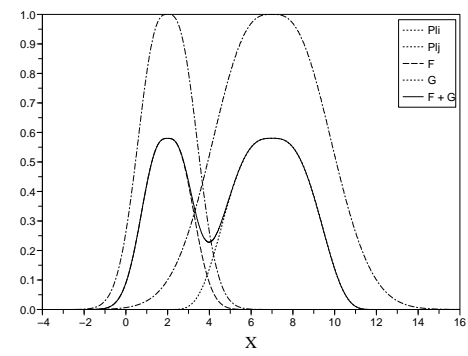

Fig. 5. Weight of conflicting intervals in the disjunctive combination.

\subsection{Global conflict}

When $n$ pieces of evidence have to be merged, the global conflict do not correspond to the sum of the partial conflicts because the domains of intervals on which they are based intersect. Consequently, the global conflict $K$ can never be lower than the most important partial conflict $k_{i, j}$ value existing between the $n$ pieces of evidence.

Depending on the pdfs characteristics, the hyper-volume's shape of conflicting elements $\left(z_{1}, \ldots, z_{n}\right)$ is complex. It is thus easier to calculate the global conflict from intervals combinations providing an non empty intersection as done in relation (17). When all supports of unimodal and consonant pdfs related to $n$ agents of evidence intersect, and if we consider $\mu_{i} \leq \mu_{j}, \forall i, j \in\{1, \ldots, n\}, i \leq j$ and to simplify, integrals infinite upper bounds assuming that $m_{i}\left(z_{i}\right)=0$ if $z_{i}>z_{\text {max }_{i}}$, the global conflict $K$ existing between these agents corresponds thus to:

$$
K=1-\int_{z_{1}=0}^{z_{1}=\infty} \int_{z_{2}=z_{M i n_{2}}}^{z_{2}=\infty} \ldots \int_{z_{n}=z_{M i n_{n}}}^{z_{n}=\infty} m_{1}\left(z_{1}\right) m_{2}\left(z_{2}\right) \ldots m_{n}\left(z_{n}\right) d z_{n} \ldots d z_{2} d z_{1}
$$

with:

$$
z_{M_{i n_{j}}}=\max \left(0, \frac{\left|\mu_{j}-\mu_{i}\right|-\Delta_{i}^{+} z_{i}}{\Delta_{j}^{-}} \forall i \in\{1, \ldots, j-1\}\right) \forall j \in\{2, \ldots, n\} .
$$


Relatively to the bounds of the considered intervals, relation (20) is equivalent to:

$$
A_{j}^{z_{M^{2 i n} j^{-}}} \leq\left(\min \left(A_{1}^{z_{1}+}, \ldots, A_{i}^{z_{i}+}\right) \forall i \in\{1, \ldots, j-1\}\right) \forall j \in\{2, \ldots, n\} .
$$

\section{Applications}

\subsection{Conjunctive and disjunction combinations of Gaussian based agents of evidence}

Under the assumption of cognitive independence, we consider three agents of evidence $\mathcal{E}_{1}, \mathcal{E}_{2}$ and $\mathcal{E}_{3}$ based on Gaussian pdfs $\mathcal{N}_{1}\left(x ; \mu_{1}=8, \sigma_{1}^{2}=4\right), \mathcal{N}_{2}\left(x ; \mu_{2}, \sigma_{2}^{2}=\right.$ $0.5)$ and $\mathcal{N}_{3}\left(x ; \mu_{3}, \sigma_{3}^{2}=1\right) . \mu_{3}$ and $\mu_{2}$ are supposed to decrease until to reach $\mu_{1}$ 's value. Partial and global conflicts calculations are given in table 5.1 when figure 6 illustrates the plausibility curves resulting of the conjunctive and disjunctive combination of these three agents of evidence.

As suggested by the plausibility curves of the conjunctive combination in figure 6 and the corresponding values of $K$, there is no linear relation between the maximum of the plausibility of this combination and the conflict. Examples illustrated in figures 6(c),6(d) and 6(e) show also the sensitivity of the conjunctive combination to the agreement of precise sources of information.

Table 1. Partial and global conflicts amounts

\begin{tabular}{l|lll|lll|l}
\hline & $\mu_{1}$ & $\mu_{2}$ & $\mu_{3}$ & $k_{1,2}$ & $k_{1,3}$ & $k_{2,3}$ & $K$ \\
\hline a) & 8 & 10.5 & 14.5 & 0.092 & 0.867 & 0.929 & 0.976 \\
b) & 8 & 10.5 & 12.5 & 0.092 & 0.451 & 0.195 & 0.522 \\
c) & 8 & 10.5 & 10.5 & 0.092 & 0.050 & 0 & 0.109 \\
d) & 8 & 10.5 & 8.5 & 0.092 & 0.000 & 0.195 & 0.257 \\
e) & 8 & 10.5 & 8 & 0.092 & 0 & 0.415 & 0.454 \\
f) & 8 & 8 & 8 & 0 & 0 & 0 & 0 \\
\hline
\end{tabular}

\subsection{Conflict management by RCR-S adaptive combination}

Many authors propose adaptive combination rules weighting conjunctive and disjunctive rules of combination. Florea et al. [6] give a general formulation of most of them and propose robust combination rules referred as RCR. To illustrate our work, we apply our relations to a RCR-S combination rule defined as:

$$
P l_{R C R-S_{1 \ldots n}}(x)=\frac{K}{1-K+K^{2}} P l_{1 @ \ldots @ n}(x)+\frac{1-K}{1-K+K^{2}} P l_{1 @ \ldots @ n}(x) .
$$

Figure 7 illustrates from left to right, the RCR-S plausibility curves obtained for the examples shown in respectively figures $6(\mathrm{~b})$ and $6(\mathrm{c})$. We observe that 


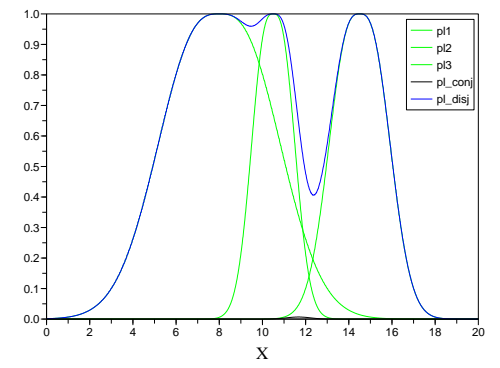

(a) $K=0.976$

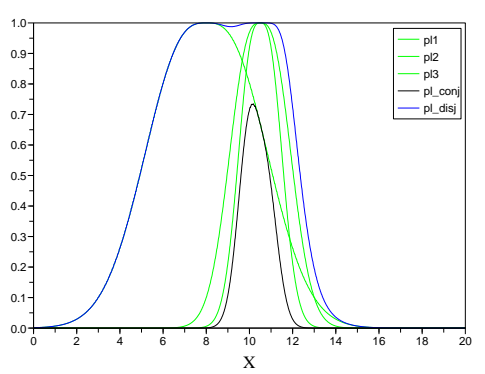

(c) $K=0.109$

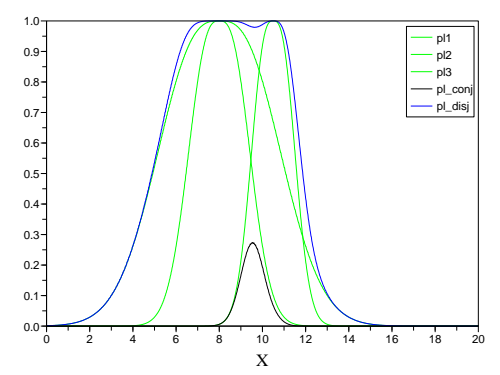

(e) $K=0.454$

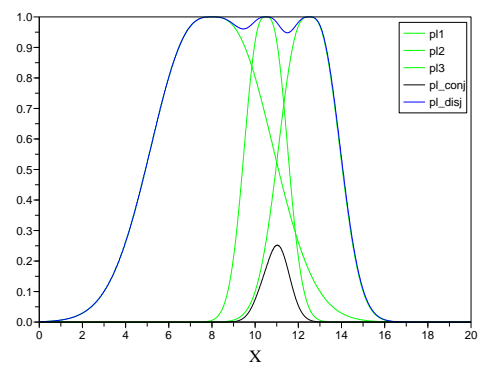

(b) $K=0.522$

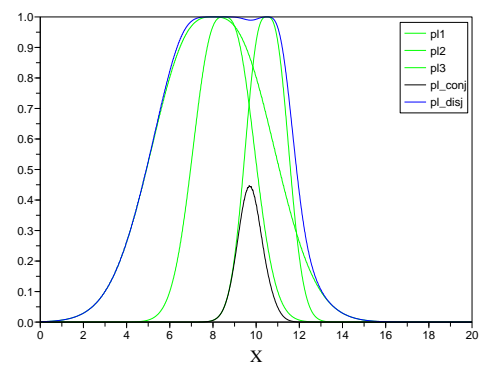

(d) $K=0.257$

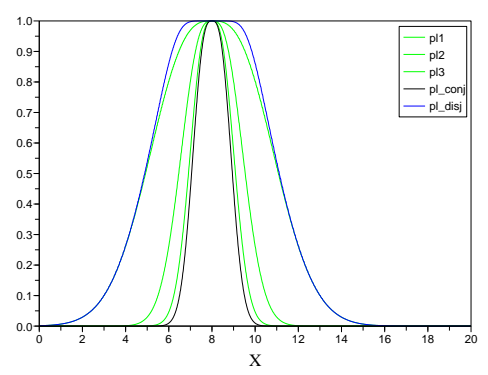

(f) $K=0$

Fig. 6. Basic combinations of 3 Gaussian based agents of evidence (plausibility curves). 
the trend in conjunctive combination is preserved even if $K>0.5$. When applied in a very high conflicting situation as illustrated in figure $6($ a) $(K=0.976)$, the $\mathrm{RCR}-\mathrm{S}$ combination operation is equivalent to the disjunctive one as expected.
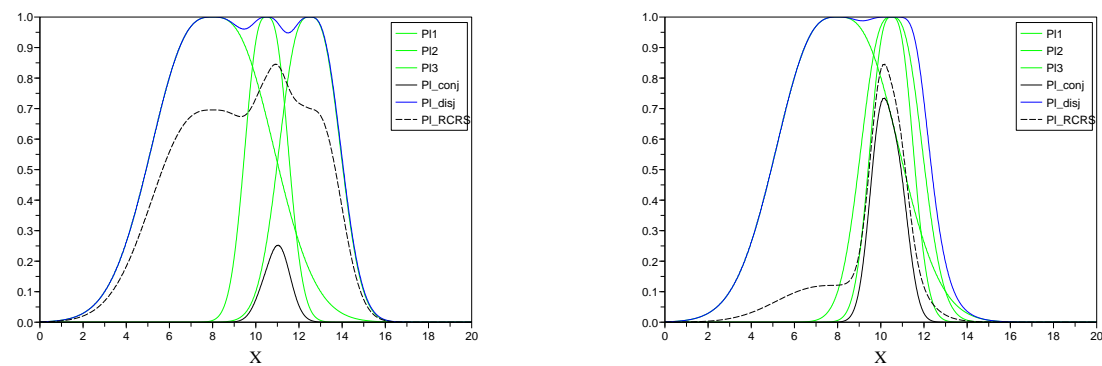

Fig. 7. Plausibilities of Conjunctive, disjunctive and RCR-S combinations.

\section{Conclusions}

Much existing adaptive combination rules can be applied on the plausibilities of conjunctive and disjunctive combinations presented here, mixed according to the global conflict value. But such a conflict's management is inefficient when one source of information is in total conflict with the other ones. Coherent sets of information sources could then be defined using partial conflicts values to perform more accurate combinations.

\section{References}

1. Smets, P.:Belief functions on real numbers. International Journal of approximate reasoning, vol. 40, no. 3, 181-223 (2005)

2. Vannobel, J.-M.:Continuous belief functions: singletons plausibility function in conjunctive and disjunctive combination operations of consonant bbds. In: Belief 2010, 6 p. (http://www.ensieta.fr/belief2010/), Brest (Fr) (2010)

3. Ristic, B., Smets,P.: Belief function theory on the continuous space with an application to model based classification. In IPMU'04, Information Processing and Management of Uncertainty in Knowledge Based Systems, pp. 1119-1126, Paris (Fr) (2004)

4. Caron, F., Ristic, B., Duflos, E., Vanheeghe, P.: Least committed basic belief density induced by a multivariate gaussian: formulation with applications. International Journal on Approximate Reasoning, vol. 48, no. 2, 419-436 (2008)

5. Smets, P.: Belief functions : the disjunctive rule of combination and the generalized Bayesian theorem. International Journal of approximate reasoning, vol.9 , no. 1., 1-35 (1993)

6. Florea, M.C., Jousselme, A.L., Bosse, E., Grenier, D.: Robust combination rules for evidence theory. Information Fusion, vol. 10, no 2, 183-197 (2009) 